\title{
Relations entre l'allongement et l'épaississement de la pousse annuelle des rejets de châtaignier (Castanea sativa Mill) au cours des deux premières années de leur développement
}

\author{
M Carlue, JM Aufort \\ Faculté des sciences, laboratoire de biologie cellulaire végétale et valorisation des espèces \\ ligneuses, 123 rue Albert Thomas, 87060 Limoges Cedex, France
}

(Reçu le 13 juin 1990; accepté le 14 février 1991)

\begin{abstract}
Résumé - L'étude a pour objectif de préciser les relations entre l'allongement et l'épaississement de la pousse annuelle au cours de 2 premières années du développement des rejets de souche chez le châtaignier. Elle est fondée sur une comparaison directe, à partir de graphiques, de l'allongement et de l'épaississement de chacune des pousses au cours de l'activité annuelle des rejets. L'évolution relative de ces deux paramètres est traduite par des courbes sur lesquelles apparaissent des "vagues" de fluctuation liées au fait qu'un type d'activité est momentanément prépondérant sur l'autre et inversement. Les rejets ont un comportement hétérogène. Le nombre de "vagues" de fluctuation varie de 1 à 4 , avec des comportements intermédiaires pour lesquels les $2^{e}$ et $3^{e}$ vagues sont seulement amorcées. Un faible nombre de rejets ne présente pas de "vagues" marquées. Les "vagues" sont d'autant plus nombreuses que la croissance de la pousse est plus importante et plus durable. La fin de la période évolutive allongement-épaississement correspond, pour un tiers des rejets, à un ralentissement progressif de la croissance en longueur alors que l'activité cambiale tend à devenir prédominante. Par contre, pour un autre tiers des rejets, l'allongement initialement prédominant s'interrompt assez brusquement, l'activité cambiale reprend alors très nettement. L'influence exercée par la souche sur la croissance des rejets est peu apparente.

Ces résultats sont discutés dans les termes de déterminisme externe ou interne du comportement des rejets, ainsi que par comparaison avec les données précédemment acquises par nous sur le même matériel.
\end{abstract}

\section{Castanea sativa / rejet / pousse annuelle / allongement / épaississement}

Summary - Relationships between elongation and diameter growth of the annual sprout during the first 2 years of development in chestnut tree stump shoots (Castanea sativa Mill). The present investigation aims to clarify the relationships between elongation and diameter growth of the annual sprout during the first 2 yr of development in chestnut tree stump shoots. The analysis is based on the direct comparison of the diagrams on lengthening and basal diameter growth of each annual sprout. The relative evolution of these 2 parameters is demonstrated by the curves on which fluctuation waves appear. They indicate that one type of growth activity prevails temporarily over the other, and conversely (figs 9-14). Stump shoot behaviour is variable; their final length and diameter are very heterogeneous (figs 1-4). The same holds true for duration of elongation activity phase (figs 5, 6). However, duration of diameter growth phase is more homogeneous (figs 7,8$)$. The number of fluctuation waves varies from 1 to 4 (figs $9,10,12,14$ ) with intermediate behavioural types in which the second and third waves are only initiated (figs 11, 13). Some stumps do not show marked fluctuation waves (fig 15). In one third of them, the '1 wave', ' 2 wave' or 'two and a half wave' stump shoots are dominated by stump shoots with only 1 elongation activity phase. On the other hand, 
most of the ' 3 wave', 'three and a half-wave' and ' 4 wave' stump shoots are dominant and their significant elongation shows 2 successive phases of activity. In another third of the stump shoots the end of the growth evolution period corresponds to a gradual decrease in the elongation speed when cambial activity tends to become predominant (figs 12, 14). Conversely, in the remaining third initially predominant elongation stops abruptly when cambial activity starts again (figs 9, 10, 15). Stump influence on the growth of shoots is low. Our results are discussed in terms of the external or internal determinism of stump shoot behaviour and also by comparison with our previous results obtained on the same material.

\section{stump / annual sprout / elongation / diameter growth}

\section{INTRODUCTION}

La croissance en longueur rythmique est une caractéristique de nombreuses espèces ligneuses tropicales. Elle se manifeste dans des conditions de milieu relativement constantes et favorables à la croissance. Des périodes d'activité intense se caractérisent alors par la formation d'entrenœuds longs; elles alternent avec des phases de croissance nulle ou très ralentie. De tels phénomènes ont été signalés chez:

- le cacaoyer (Greenwood et Posnette, 1950; Greathouse et al, 1971; Vogel, 1975);

- le manguier (Holdsworth, 1963; Scarrone 1969);

- l'hévéa (Halle et Martin, 1968);

- le Gnetum africanum (Mialoundama, 1985).

Des comportements de même nature se manifestent chez des espèces ligneuses de climat tempéré cultivées en conditions constantes en chambre climatisée, en particulier dans le cas du chêne (Payan, 1982), du hêtre (Klebs, 1914; Lavarenne et al, 1971) et du châtaignier ( $\mathrm{Si}$ Mohamed, 1983). Une telle rythmicité de la croissance en longueur peut également s'observer dans la nature sous nos climats. Elle se manifeste alors sous la forme des pousses herbacées du Lilas (Klebs, 1914) ou des pousses de la Saint-
Jean du hêtre, du chêne ou de divers arbres fruitiers (Champagnat et al, 1961; Lavarenne et al, 1971).

Les travaux qui abordent le problème des rapports entre l'allongement et l'épaississement au cours de la croissance rythmique sont rares. Chez l'Hevea brasiliensis (De Fay, 1986) l'activité cambiale est périodique, elle semble sous la dépendance du rythme de la croissance apicale et en phase avec lui. Dans le cas du chêne (Payan, 1982; Champagnat et al, 1986) la croissance en épaisseur de la tige est aussi périodique mais elle se manifeste en alternance de phase avec son allongement. Ces résultats posent le problème de l'origine de la rythmicité. L'activité cambiale est-elle le facteur prépondérant ou alors est-ce la xylogenèse qui est déterminante (alternance de la formation des vaisseaux et des fibres) ?

Nos observations en conditions naturelles, sur les rejets de souche du châtaignier (Aufort et al, 1990), ont mis en évidence la difficulté d'analyser clairement les résultats comparés de l'allongement et de l'épaississement. Les variations de croissance en longueur et en diamètre ont été représentées chacune en fonction du temps et sur des graphiques ne prenant en compte que des valeurs différentielles. II apparaît alors que :

- la croissance en longueur des pousses s'établit le plus souvent en 2 phases principales au cours de la période d'activité an- 
nuelle. L'épaississement des pousses se manifeste également irrégulièrement, mais sans phases nettement marquées;

- une relation linéaire significative s'établit entre le diamètre final à la base de la pousse annuelle des rejets et la longueur finale de cette pousse;

- les valeurs moyennes hebdomadaires de la température sont le plus souvent bien corrélées avec l'allongement, mais elles ne le sont jamais avec l'épaississement.

II existe donc une ambiguïté fondamentale dans les relations liant l'allongement et l'épaississement des rejets. La méthode d'analyse que nous nous proposons d'appliquer consiste à considérer les valeurs cumulées de l'allongement et de l'épaississement et de les associer en couples de données ( $x=$ allongement, $y=$ épaississement) sur un même graphique. Elle doit permettre de préciser la nature des relations liant la croissance en longueur et la croissance en épaisseur et en particulier de fournir des éléments de réponse à une question fondamentale se posant à leur propos :

- le comportement individuel des rejets est-il à cet égard homogène ou au contraire caractérisé par une variabilité clairement descriptible et dont on pourra discuter les divers aspects et la causalité.

\section{MATÉRIEL ET MÉTHODES}

La parcelle de taillis de châtaignier utilisée se trouve sur la commune de Châlus, dans le sud ouest du département de la Haute-Vienne. Cette région de collines, s'élevant jusqu'à une altitude d'environ $550 \mathrm{~m}$, jouit d'un climat à tendance océanique. Les précipitations sont abondantes, de l'ordre de $1200 \mathrm{~mm}$ par an en moyenne, sans saison sèche marquée (Vilks, 1974).

Les observations débutent en mai et s'achèvent en octobre 1983. Elles permettent d'appré- cier la croissance des pousses qui se développent sur des souches recépées au cours de l'hiver précédent (pousse de première année) et sur des rejets d'un an (pousse de deuxième année).

\section{Évaluation de la croissance des pousses}

La croissance des pousses est appréciée selon 2 critères : allongement et augmentation du diamètre des pousses.

L'intervalle de temps séparant 2 séries d'observations est d'une semaine. Les observations sont effectuées à heures régulières d'une semaine sur l'autre dans le but de limiter l'incidence des variations de turgescence des cellules cambiales lors de l'estimation du diamètre des rejets.

Les mesures sont réaiisées sur un échantillon comprenant 25 pousses. Elles sont réparties sur 5 souches. Les 5 rejets choisis sont portés par des secteurs différents de la souche. Les longueurs sont mesurées à l'aide d'un mètre souple avec une précision de l'ordre du $\mathrm{cm}$. Le diamètre est pris à la base de la pousse. On utilise un pied à coulisse électronique qui donne $0,1 \mathrm{~mm}$. Malgré les précautions prises (repérage du niveau de mesure, orientation de l'appareil) la précision n'est sans doute pas supérieure à $0,5 \mathrm{~mm}$.

\section{Traitement des données numériques et présentation des résultats}

Pour l'étude de la croissance de la pousse de première année, il n'est pas possible, au départ, de distinguer des rejets selon des classes de vigueur, car on ne connait pas le devenir des bourgeons qui se développent sur la souche. Le choix est donc tout à fait aléatoire.

Pour l'étude des pousses de deuxième année, nous avons donc été conduits à utiliser également un échantillon non homogène. Cependant, comme les brins les plus chétifs meurent très rapidement, ils ont été écartés. Ceux qui ont été choisis atteignaient au moins $1,30 \mathrm{~m}$ de longueur à la fin de la première année de croissance. 
Les résultats concernant les relations entre l'allongement et l'épaississement des pousses sont analysés pour chacun des rejets. Nous regroupons sur un graphique les couples de valeurs correspondant à l'allongement et à l'épaississement d'un rejet (abscisse : allongement, ordonnée : épaississement). Cette méthode présente un avantage déterminant pour l'analyse des relations allongement-épaississement : elle permet l'obtention de graphiques à partir desquels les fluctuations relatives de l'allongement et de l'épaississement des rejets apparaissent sous la forme de "vagues" sur les courbes. Ces dernières peuvent être mises très clairement en évidence et traduisent le fait qu'un type d'activité de croissance puisse être prédominant sur l'autre ou inversement.

\section{RÉSULTATS}

\section{Aspects généraux de la croissance des rejets}

\section{Taille de la pousse annuelle des rejets}

Au 4 mai, date du début des observations, la croissance est déjà amorcée pour toutes les pousses. Leur longueur est comprise entre 2 et $12 \mathrm{~cm}$ la $1^{\text {re }}$ année et entre 5 et $13 \mathrm{~cm}$ la $2^{e}$ année.

À la fin de leur croissance en longueur, les pousses de $1^{\text {re }}$ année mesurent entre 33 et $208 \mathrm{~cm}$ (fig 1). La taille des pousses de $2^{\mathrm{e}}$ année est comprise entre 26 et 149 $\mathrm{cm}$ (fig 2). La distribution de la longueur finale des pousses de $1^{\text {re }}$ année est assez homogène : la majorité d'entre elles mesurent entre 150 et $200 \mathrm{~cm}$ (fig 1). La distribution n'est pas homogène la $2^{\mathrm{e}}$ année et les pousses sont plus courtes (fig 2).

L'épaisseur finale des pousses est comprise entre 8 et $26 \mathrm{~mm}$ la $1^{\text {re }}$ année (fig 3) et entre 7,5 et $22 \mathrm{~mm}$ la $2^{\mathrm{e}}$ année (fig 4). La distribution est assez homogène dans les 2 cas. II existe une majorité de pousses dont le diamètre est compris
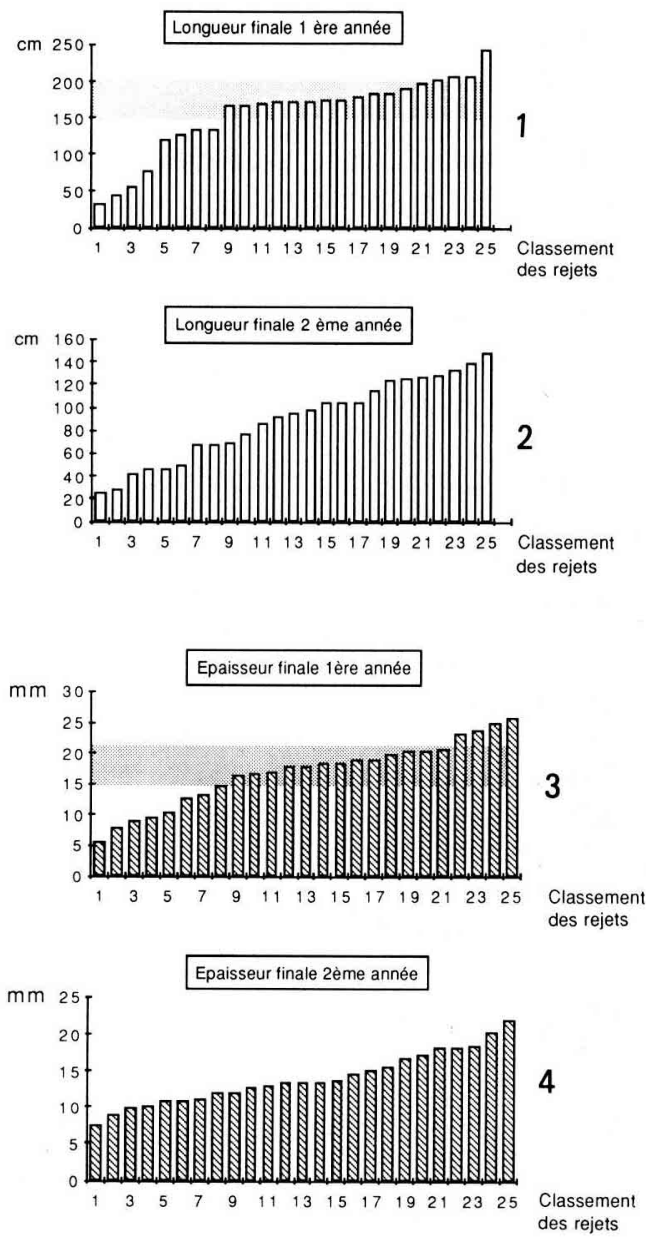

Figs 1-4. 1. Distribution de la longueur finale des rejets de $1^{\text {re }}$ année. Le classement des rejets est effectué par ordre de taille croissante sur l'axe des abscisses. 2. Distribution de la longueur finale des rejets de $2^{\theta}$ année. Le classement des rejets est effectué par ordre de taille croissante sur l'axe des abscisses. 3. Distribution de l'épaisseur finale des rejets de $1^{\text {re }}$ année. Le classement des rejets est effectué par ordre de taille croissante sur l'axe des abscisses. 4. Distribution de l'épaisseur finale des rejets de $2^{\theta}$ année. Le classement des rejets est effectué par ordre de taille croissante sur l'axe des abscisses. 
entre 15 et $20 \mathrm{~mm}$ la $1^{\text {re }}$ année et entre 10 et $15 \mathrm{~mm}$ la $2^{\mathrm{e}}$ année.

\section{Durée de la croissance des pousses}

La durée de la croissance en longueur des pousses de $1^{\text {re }}$ année est variable. L'allongement peut s'arrêter précocement au 15 juin alors que certaines pousses prolongent leur croissance jusqu'au 21 septembre (fig 5), 24\% d'entre elles stoppent leur croissance au 31 août. La durée de la période d'allongement est également variable la $2^{\mathrm{e}}$ année. L'arrêt de l'allongement apparaît du 8 juin au 24 août (fig 6), 24\% des rejets stoppent leur croissance au 3 août. Un grand nombre de pousses de tre $^{\mathrm{r}}$ et de $2^{\mathrm{e}}$ année manifestent une période d'arrêt momentané ou de ralentissement de croissance en longueur, de la fin juin au début juillet.

La durée de la croissance en épaisseur des pousses de $1^{\text {re }}$ année est variable (fig 7). L'épaississement peut s'arrêter précocement au 15 juin alors que $56 \%$ des pousses prolongent leur croissance diamétrale jusqu'au 21 septembre. Une variabilité identique apparaît la $2^{\mathrm{e}}$ année (fig 8), l'arrêt de l'épaississement s'échelonne du 20 juillet au 14 septembre, $33 \%$ des pousses prolongent leur croissance en épaisseur jusqu'à la mi-septembre. L'épaississement se prolonge après l'arrêt de l'allongement pour $92 \%$ des pousses de $1^{\mathrm{re}}$ et $2^{\mathrm{e}}$ année (Aufort et al, 1990). L'arrêt simultané de l'allongement et de l'épaississement ne concerne que $8 \%$ d'entre elles.

\section{Aspect des relations allongement-épaississement}

Plusieurs catégories de comportement des rejets sont mises en évidence au cours de leur pousse annuelle.
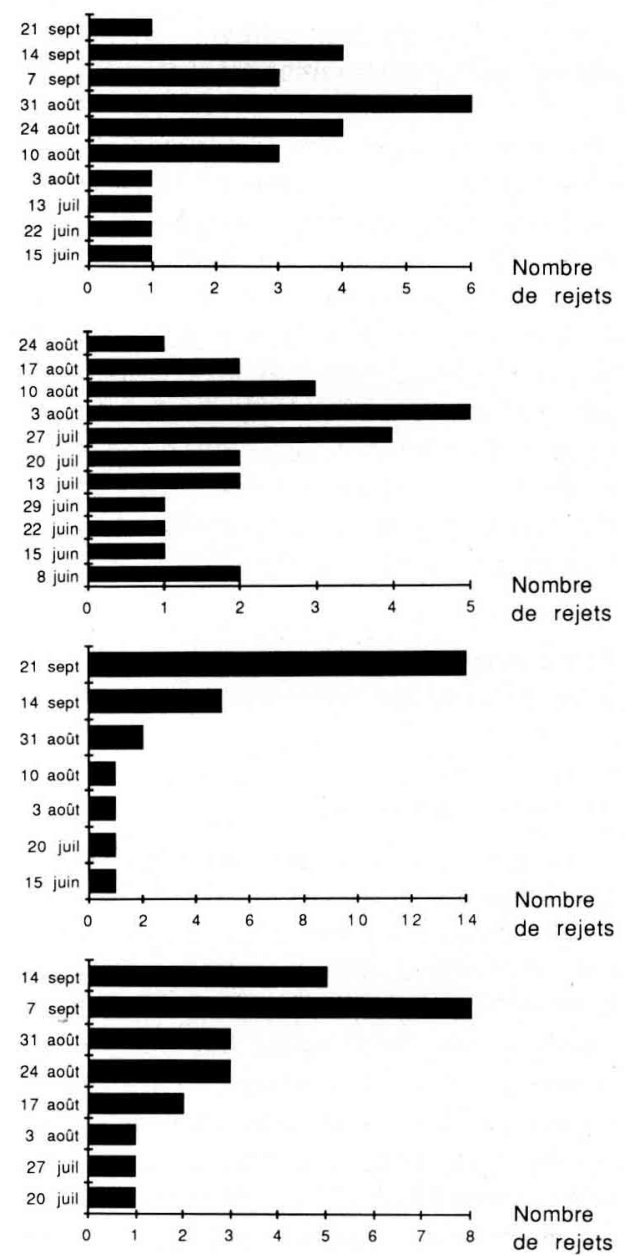

Figs 5-8. 5. Distribution du nombre de rejets de 1re année en fonction de la date d'arrêt de leur croissance en longueur. En ordonnée, durée de l'allongement $1^{\text {re }}$ année. 6. Distribution du nombre de rejets de $2^{e}$ année en fonction de la date d'arrêt de leur croissance en longueur. En ordonnée, durée de l'allongement $2^{\theta}$ année. 7. Distribution du nombre de rejets de $1^{\text {re }}$ année en fonction de la date d'arrêt de leur croissance en épaisseur. En ordonnée, durée de l'épaississement $1^{\text {re }}$ année. 8. Distribution du nombre de rejets de $2^{\mathrm{e}}$ année en fonction de la date d'arrêt de leur croissance en épaisseur. En ordonnée, durée de l'épaississement $2^{\mathrm{e}}$ année. 


\section{Une "vague» de fluctuation allongement-épaississement (fig 9)}

Un seul cas peut être observé pour les pousses de $1^{\text {re }}$ année alors que 6 pousses de $2^{\mathrm{e}}$ année présentent ce type de comportement. Cinq de ces dernières sont courtes (respectivement 26, 43, 47, 47 et $50 \mathrm{~cm}$ ), leur croissance en longueur s'interrompt précocement ( 8 juin, 8 juin, 15 juin, 22 juin, 29 juin) et la durée de leur période d'allongement varie de 5 à 8 semaines. La "vague" de fluctuation apparaît précocement et se manifeste du 4 au 25 mai pour la plupart des rejets.

\section{Deux "vagues" de fluctuation allongement-épaississement (fig 10)}

Cinq cas peuvent être observés la $1^{\text {re }}$ année et 4 la $2^{e}$ année.

La longueur finale des pousses de $1^{\text {re }}$ année est très variable (de 33 à $200 \mathrm{~cm}$ ), leur croissance s'interrompt à des dates très différentes (du 15 juin au 14 septembre). La durée de la période d'allongement est très hétérogène (de 6 à 19 semaines). La variabilité est moins accentuée dans le cas de pousses de $2^{e}$ année. Les longueurs finales sont comprises entre 68 et $129 \mathrm{~cm}$, les dates d'arrêt de croissance s'échelonnent du 13 juillet au 17 août et les durées des périodes d'allongement fluctuent entre 10 et 15 semaines.

La $1^{\text {re }}$ "vague» peut apparaître plus ou moins précocement, du début mai à la mimai dans les cas les plus précoces, de la mi-mai au début juin dans les cas les plus tardifs. La $2^{\mathrm{e}}$ "vague» de croissance est souvent moins accentuée que la première. Elle débute au bout de 5-10 semaines de croissance, entre la $1^{\text {er }}$ juin et le 6 juillet.

\section{Deux «vagues» de fluctuation allongement-épaississement et amorce d'une $3^{e}$ "vague» (fig 11).}

Trois pousses de $1^{\text {re }}$ année et 3 pousses de $2^{\mathrm{e}}$ année manifestent ce type de com-
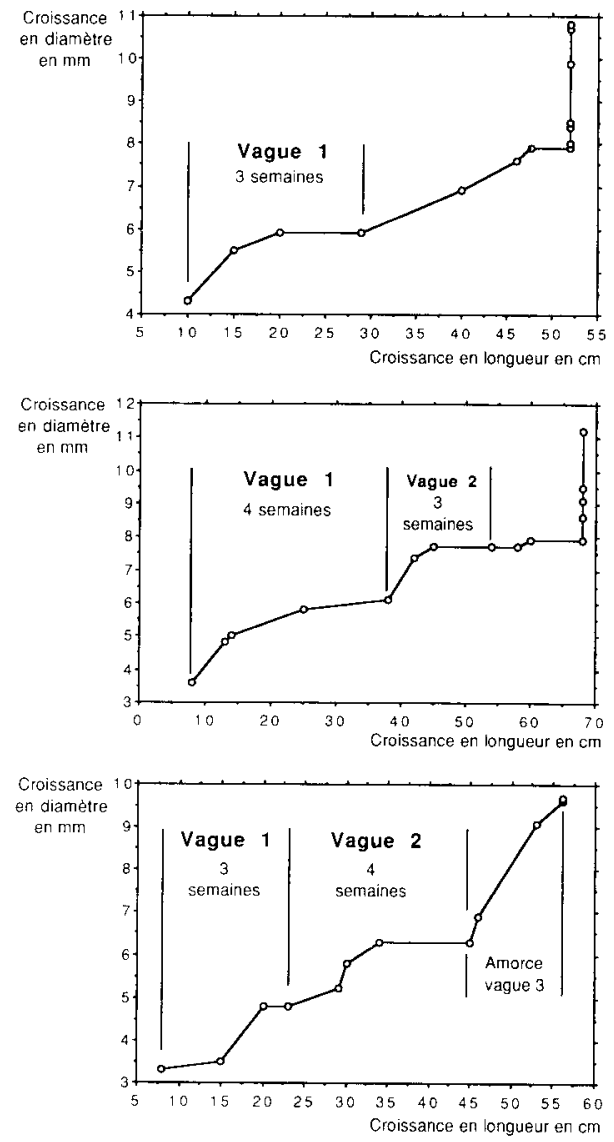

Figs 9-11. 9. Relation allongement-épaississement pour un rejet de $2^{\mathrm{e}}$ année. Cas d'une seule "vague" de fluctuations. 10. Relation allongement-épaississement pour un rejet de $2^{\mathrm{e}}$ année. Cas de deux "vagues» de fluctuations. 11. Relation allongement-épaississement pour un rejet de $1^{\text {re }}$ année. Cas de deux "vagues" de fluctuations suivies par l'amorce d'une $3^{\theta}$ "vague". 
portement. Les tailles des pousses de $1^{\text {re }}$ année sont peu homogènes (1 pousse courte : $56 \mathrm{~cm}$ et 2 pousses longues : 173 et $179 \mathrm{~cm}$ ). Celles des pousses de $2^{e}$ année le sont beaucoup plus (entre 96 et $133 \mathrm{~cm}$ ). La durée de l'allongement des pousses de $1^{\text {re }}$ année est assez variable (de 10 à 17 semaines). Les interruptions de leur croissance en longueur se manifestent de la mi-juillet à la fin du mois d'août (de 10 à 17 semaines de croissance). Celles des pousses de $2^{e}$ année sont assez synchrones et apparaissent du 3 août au 10 août (de 13 à 14 semaines de croissance).

L'amorce de la $3^{e}$ "vague" des pousses de $1^{\text {re }}$ année apparaît au 3 et au 10 août lorsque leur croissance est prolongée et au 22 juin lorsque leur croissance est brève. Elle se manifeste au 6 juillet et au 13 juillet la $2^{e}$ année. Cette amorce est la conséquence d'une reprise de l'activité d'épaississement de la pousse lors de la phase ultime de sa croissance. Elle précède l'arrêt définitif de son allongement.

\section{Trois "vagues" de fluctuation allongement-épaississement (fig 12)}

Dix pousses de $1^{\text {re }}$ année et 4 pousses de $2^{\mathrm{e}}$ année entrent dans cette catégorie. Sept des 10 pousses de $1^{r e}$ année atteignent une grande taille finale $(169,176$, 186, 193, 208, 208 et $245 \mathrm{~cm}$ ). Trois pousses sur 10 sont par contre nettement plus courtes en fin de croissance $(79,122$ et $135 \mathrm{~cm}$ ). Les durées des périodes de croissance des 7 pousses les plus longues sont homogènes (de 17 à 20 semaines de croissance). Elles caractérisent des pousses à croissance en longueur prolongée (arrêt de l'allongement tardif de la fin août à la mi-septembre). Les 4 pousses de $1^{\text {re }}$ année sont de taille moyenne $\{87,93$, 104 et $104 \mathrm{~cm}$ ). L'arrêt de leur croissance est assez précoce (du 27 juillet au 3 août) et la durée de la période d'allongement est de 12 ou 13 semaines.

La $3^{e}$ "vague" de fluctuation allongement-épaississement ne se met pas en place simultanément pour les 10 pousses de $1^{\text {re }}$ année. Elle débute aux 13 juillet, 20 juillet, 27 juillet et 3 août. La fin de cette $3^{e}$ "vague» apparaît du 3 au 17 août, lorsque la croissance en longueur de la pousse n'évolue plus. Comme pour les pousses de $1^{\text {re }}$ année, sa mise en place n'est pas simultanée pour les 4 pousses de $2^{e}$ année. Elle s'établit aux 22, 29 juin, 6 et 13 juillet et s'achève entre le 6 et le 27 juillet.

\section{Trois "vagues» de fluctuation allongement-épaississement et amorce d'une $4^{e}$ "vague» (fig 13)}

Ce comportement apparaît pour 3 pousses de $1^{\text {re }}$ année. Deux d'entre elles sont des pousses longues (184 et $204 \mathrm{~cm}$ ) alors que le $3^{e}$ est de taille moyenne $(127 \mathrm{~cm})$. La durée de leur période d'allongement est respectivement de 14,16 et 18 semaines, ce qui correspond à des arrêts de croissance en longueur aux 10 août, 24 août et 7 septembre. Le début de cette $3^{e}$ "vague" apparaît respectivement aux 13 juillet, 3 et 10 août pour chacune de ses 3 pousses, c 'est-à-dire au bout de 10,13 et 14 semaines de croissance.

L'amorce de cette $4^{e}$ "vague" est la conséquence d'une reprise de l'activité d'épaississement de la pousse lors de la phase ultime de sa croissance. Elle précède l'arrêt définitif de son allongement.

\section{Quatre "vagues" de fluctuation allongement-épaississement (fig 14)}

Ce comportement apparaît pour une pousse de $1^{\text {re }}$ année et 2 pousses de $2^{\mathrm{e}}$ année. La pousse de première année est assez longue $(173 \mathrm{~cm})$. La durée de sa pé- 

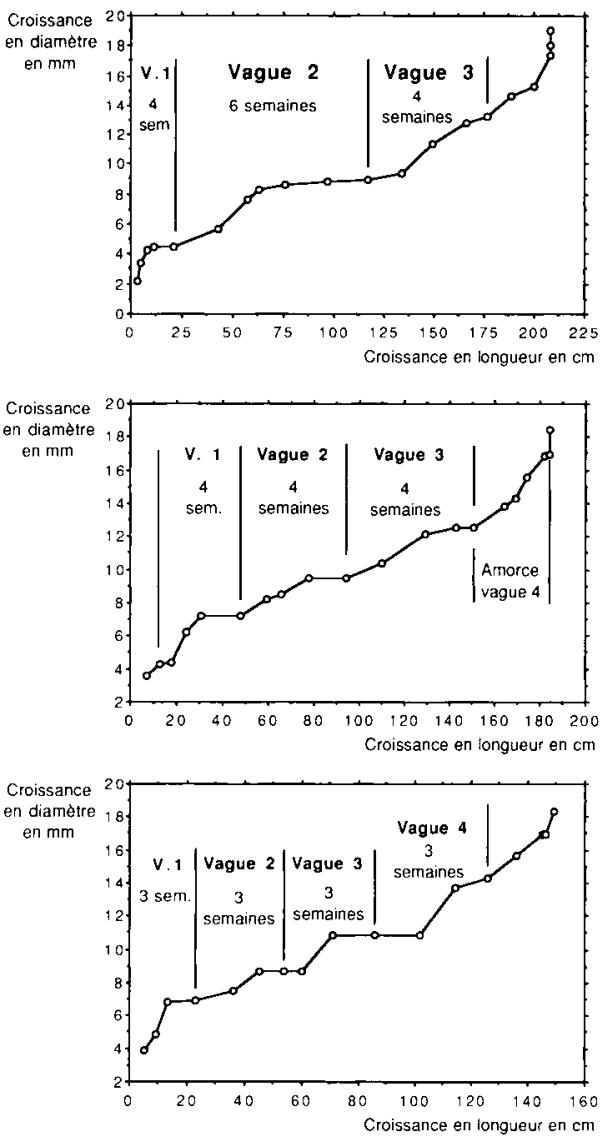

Figs 12-14. 12. Relation allongement-épaississement pour un rejet de $1^{\text {re }}$ année. Cas de 3 "vagues" de fluctuations. 13. Relation allongement-épaississement pour un rejet de $1^{\text {re }}$ année. Cas de 3 "vagues de fluctuations suivies par l'amorce d'une $4^{\theta}$ "vague". 14. Relation allongement-épaississement pour un rejet de $2^{\mathrm{e}}$ année. Cas de 4 "vagues" de fluctuations.

riode de croissance est de 16 semaines, elle correspond à un arrêt de croissance en longueur au 17 août. Les pousses de deuxième année ont des longueurs finales respectives de 140 et $149 \mathrm{~cm}$, elles sont relativement élevées pour l'échantillon. La durée de leur période de croissance est de 15 et 16 semaines, l'allongement des pousses s'achève aux 17 et 24 août.

La 4e "vague" de croissance des pousses de première année se met en place au 3 août. Elle apparaît plus précocement pour les pousses de $2^{\mathrm{e}}$ année (aux 13 et 20 juillet).

\section{Cas particulier des pousses sans "vagues" de fluctuation allongement-épaississement nettement marquées (fig 15)}

Ce comportement apparaît pour 2 pousses de $1^{\text {re }}$ année et 2 pousses de $2^{\mathrm{e}}$ année. Les longueurs finales des pousses de tre $^{\mathrm{r}}$ année sont de 134 et $176 \mathrm{~cm}$. Les durées de leurs périodes de croissance en longueur sont respectivement de 16 et 17 semaines. L'arrêt de l'allongement apparaît aux 24 et 31 août. Les longueurs finales des pousses de $2^{\mathrm{e}}$ année atteignent des valeurs qui s'étagent entre 68 à $176 \mathrm{~cm}$. Les durées des périodes des croissance en longueur sont comprises entre 11 et 17 semaines. Chacune des pousses est caractérisée par une reprise très nette de la croissance en épaisseur, en fin de période de croissance en longueur. Cette reprise s'effectue au 27 juillet pour les pousses de $1^{r e}$ année et au 6 juillet pour celles de $2^{e}$ année.

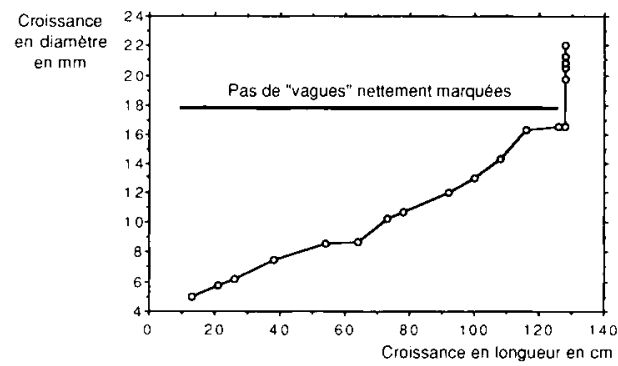

Fig 15. Relation allongement-épaississement pour un rejet de $2^{e}$ année. Absence de "vagues" nettement caractérisées. 


\section{DISCUSSION ET CONCLUSION}

L'aspect des courbes s'explique par les fluctuations relatives des activités d'allongement et d'épaississement

Un type d'activité de croissance prédomine momentanément sur l'autre et inversement. Notre analyse, dans le cas du châtaignier, montre une succession de "vagues de fluctuations". Si nous nous référons à l'aspect le plus souvent présenté pour la première d'entre elles, l'épaississement est d'abord favorisé, puis l'allongement. Nos résultats sont à rapprocher de ceux de Payan (1982), obtenus sur Quercus pedunculata. En effet, l'augmentation en diamètre de la tige des plantules de chêne est décalée et en avance d'un quart de phase sur son allongement. De plus, dans ce cas, le rythme d'accroissement en épaisseur a une période comprise entre 20 et $35 \mathrm{j}$ alors que les vagues de fluctuation allongement-épaississement s'étendent également sur plusieurs semaines (entre 14 et 56 jours) pour le châtaignier.

Cependant, les méthodes expérimentales utilisées dans l'un et l'autre cas sont différentes et incitent à une comparaison prudente des résultats. Les plantules de chêne étaient cultivées en conditions contrôlées. Elles étaient observées tous les 2 j et les données étaient regroupées en valeurs moyennes. Notre travail est, par contre, réalisé en conditions naturelles. Les observations sont effectuées toutes les 2 semaines et les résultats sont exprimés en valeurs individuelles.

L'analyse individuelle permet de mettre en évidence un comportement hétérogène des rejets. Cette hétérogénéité est d'abord caractérisée par une variation du nombre de "vagues" de fluctuation allongementépaississement (de 1 à 4 "vagues"). Nous relevons deux comportement dominants :
- rejets avec 2 «vagues» de fluctuation (12 cas);

- rejets avec 3 «vagues» de fluctuation (17 cas).

L'hétérogénéité du comportement des rejets se rapporte également à l'aspect de la phase finale de croissance, 2 cas peuvent être distingués :

- un ralentissement progressif de l'allongement se manifeste et conduit à une prépondérance de l'épaississement pour 15 rejets (figs 12 et 14). L'activité cambiale persistera après l'arrêt de la croissance en longueur. Ce résultat est en conformité avec le fait que, sur notre matériel, $90 \%$ des rejets prolongent leur activité cambiale bien après l'arrêt de l'allongement (Aufort et al, 1990). II confirme que, sur ce point, le châtaignier se comporte comme beaucoup d'autres espèces ligneuses (Wareing et Roberts, 1956);

- la croissance en longueur peut devenir nettement prédominante sur l'épaississement. Cette phase est aussitôt suivie par un net arrêt de l'allongement accompagné d'une reprise simultanée de l'activité d'épaississement, 16 rejets sont dans ce cas (figs 9 et 10). Ce résultat apparaît donc fondamentalement différent du précédent. Dans ce cas, l'activité des méristèmes primaires ne s'interrompt pas progressivement mais au contraire assez brusquement. Si les arrêts de croissance des rejets apparaissaient simultanément, on pourrait expliquer ce résultat par l'influence inhibitrice d'un facteur de l'environnement. Le raccourcissement des jours est par exemple un facteur inducteur de l'arrêt de la croissance en longueur des arbres (Lavender, 1980), surtout dans le cas où ils présentent, comme dans le cas du châtaignier (Aufort et al, 1990), un allongement prolongé s'établissant par "vagues" successives (Heide, 1974). Mais les arrêts de croissance de ces rejets ne sont pas 
synchrones. Ils apparaissent entre 8 à 18 semaines de croissance pour les rejets d' 1 an et entre 6 à 14 semaines de croissance pour ceux de 2 ans. L'hypothèse d'un déterminisme externe commun à l'arrêt de la croissance en longueur de tous ces rejets est donc à rejeter.

Dans le cas des rejets à faible croissance (rejets dominés), l'interruption brutale de l'allongement apparaît fin juin ou début juillet. Elle pourrait être le résultat d'une compétition trophique entre les rejets portés par une même souche. Les plus grosses racines de la cépée de châtaignier sont caractérisées par un fort potentiel de stockage, ce qui permet de les considérer comme des organes de réserve, contrairement aux souches (Dubroca, 1983). La proximité ou l'éloignement des jeunes rejets par rapport à ces racines peut expliquer la plus ou moins grande disponibilité des réserves à leur profit et par conséquent les arrêts précoces et assez brutaux de la croissance en longueur de certains rejets. D'autre part, le vieillissement des taillis de châtaignier que nous étudions peut également constituer une des causes explicatives de cet arrêt précoce de la croissance des rejets. Au cours du temps, une cépée et son ensouchement se scindent en plusieurs sousunités dotées chacune de leur système racinaire propre. Des variations dans le fonctionnement de ces systèmes peuvent rendre compte des différences de développement des rejets de la cépée étudiée (Aymard et Fredon, 1986).

Dans le cas des rejets à croissance importante (rejets dominants), l'interruption brutale de l'allongement apparaît au mois d'août. Le raccourcissement des jours pourrait alors l'expliquer (Heide, 1974; Lavender, 1980), d'autant mieux que le châtaignier, comme dans le cas des espèces ligneuses à forte sensibilité photopériodique (Nitsch, 1957), subit une abscission de son bourgeon terminal en août (Aufort et al, 1980). Toutefois cette hypothèse explicative fondée sur l'influence d'un seul facteur externe est trop limitée, la réponse des ligneux à la photopériode peut être modifiée par d'autres facteurs de l'environnement comme la teneur en eau du sol et sa température (Kramer et Kozlowski, 1979).

L'hétérogénéité du comportement des rejets est-elle plus ou moins superposable avec celle mise en évidence lors de l'étude dynamique de la croissance des rejets (Aufort et al, 1990) ?

- Les rejets à 1 "vague", 2 "vagues" et 2,5 "vagues" de fluctuations allongementépaississement sont, pour un tiers d'entre eux, des rejets dominés dont l'allongement est souvent réduit à une seule phase d'activité stoppée précocement (tailles finales comprises entre 26 et $70 \mathrm{~cm}$ et arrêt de l'allongement entre le 8 juin et le 20 juillet). Les deux tiers des rejets restants ont une croissance en longueur nettement plus importante (tailles finales comprises entre 77 et $200 \mathrm{~cm}$ ). Cette croissance se présente en 2 périodes d'activité séparées par un ralentissement ou par un arrêt momentané entre le 22 juin et le 6 juillet.

- Par contre, la plupart de rejets à 3 "vagues", 3,5 "vagues" et 4 "vagues" de fluctuation sont des rejets dominants dont la croissance importante et prolongée (tailles finales comprises entre 79 et 245 $\mathrm{cm}$ et arrêts de la croissance en longueur situés entre le 3 août et le 21 septembre) se présente en 2 phases d'activité.

- Il apparaît donc une relation significative entre le nombre de "vagues" de fluctuation et la taille finale des rejets. Les "vagues" sont d'autant plus nombreuses que la croissance du rejet est plus importante et plus durable. Cette constatation justifie l'idée selon laquelle l'évolution des relations allongement-épaississement se 
manifeste de façon cyclique, le nombre de "phases" du cycle (nombre de "vagues") étant fonction de la longueur finale du rejet et de la durée de sa période de croissance.

La souche peut-elle influencer l'évolution des rapports allongementépaississement?

II s'avère en effet que, dans le cas des rejets de $1^{\text {re }}$ année, 4 rejets d'une même souche se comportent de manière assez identique, 2 "vagues" de fluctuation apparaissent, elles sont suivies dans un cas par l'amorce d'une troisième "vague". Cependant, ce résultat doit être nuancé : le choix des rejets observés la $1^{\text {re }}$ année est aléatoire car on ne connait pas le devenir des bourgeons qui se développent sur la souche. En fait, les comportements des 4 rejets de cette souche sont assez voisins (longueurs finales comprises entre 122 et $193 \mathrm{~cm}$, épaisseurs finales variant de 16,4 à $25 \mathrm{~mm}$ et durée de période de croissance s'étendant de 14 à 18 semaines). II est donc possible que cette homogénéité apparente dans l'évolution des relations allongement-épaississement soit due au hasard de l'échantillonnage. L'hypothèse d'un «effet souche» ne pourrait être vérifiée que par une étude du comportement de tous les rejets portés par chacune d'entre elles. D'autre part, la notion de "souche» est toujours discutable sur des taillis vieillissants. Elle correspond en fait au regroupement de plusieurs sous-unités assez nettement individualisées (Aymard et Fredon, 1986).

II conviendrait d'approfondir l'analyse afin de préciser le déterminisme de la succession des "vagues" de fluctuation allongement-épaississement pendant la période de végétation annuelle. Les résultats obtenus sur d'autres espèces ligneuses, en particulier sur le chêne, permettent d'orienter les futures recherches.

La périodicité de la croissance et plus particulièrement celle de l'activité cambiale est conditionnée par la température, la photopériode et l'approvisionnement en eau (Philipson et al, 1971; Kramer et Kozlowski, 1979). Une étude écophysiologique de la croissance des rejets devrait donc permettre de mieux distinguer la part des causes externes et celle du déterminisme endogène dans ces phénomènes périodiques.

D'autre part, le rythme de la croissance en longueur et celui de l'activité cambiale sont souvent subordonnés à la croissance foliaire (Brown, 1971; Payan, 1982). L'observation de la dynamique de formation et d'étalement des feuilles ainsi que la pratique de leur ablation peuvent donc apporter des informations complémentaires sur la nature du déterminisme interne de ces "vagues" de croissance.

Diverses interprétations sont émises actuellement pour rendre compte des phénomènes de la croissance rythmique chez les ligneux, même si elles ne sont pas avancées pour le moment à propos du rythme de la croissance en épaisseur. Sur Quercus palustris (Borchert, 1975), le rythme de croissance de la pousse peut être attribué à un déficit hydrique interne résultant d'un déséquilibre entre tige et racine. Des études récentes réalisées sur Quercus pedunculata (Barnola et al, 1986; Champagnat, 1989; Alatou et al, 1989; Barnola et al, 1990) font apparaître que le rythme de croissance des jeunes plants peut être attribué à une fluctuation entre 2 états physiologiques différents dans le bourgeon apical. Elle met en cause le métabolisme des nucléotides adényliques et non adényliques ainsi que les propriétés membranaires. Le déterminisme de la croissance rythmique serait lié aux variations régulières et fugaces des possibilités d'échange entre le bourgeon terminal, les tissus sous-jacents et les jeunes feuilles. Ces résultats amènent à se demander si le décalage entre allongement et épaississe- 
ment observé sur les rejets de châtaignier ne résulterait pas d'une concurrence entre 2 zones consommatrices (méristème apical et cambium) recevant un flux nutritif limité ?

\section{REMERCIEMENTS}

Les auteurs adressent leurs vifs remerciements à S Lachaud, maître de conférences au Laboratoire de physiologie et biochimie végétales de l'université de Poitiers, pour ses critiques et suggestions.

\section{RÉFÉRENCES}

Alatou D, Barnola $P$, Lavarenne, S, Gendraud M (1989) Caractérisation de la croissance rythmique du Chêne pédonculé. Plant Physiol Biochem 27, 275-280

Aufort JM, Carlue M, Fredon JJ (1990) Croissance de la pousse annuelle au cours des deux premières années du développement des rejets de châtaignier (Castanea sativa Mill). Mise en évidence d'un rythme endogène, influence du facteur thermique. Ann Sci For 47, 241-253

Aymard M, Fredon JJ (1986) Étude des relations entre une racine et les rejets de la souche chez Castanea sativa Mill. Ann Sci For 43, 351-364

Barnola P, Crochet A, Payan E, Gendraud M, Lavarenne $S$, (1986) Modifications du métabolisme énergétique et de la perméabilité dans le bourgeon apical et l'axe sous-jacent au cours de l'arrêt de croissance momentané de jeunes plants de chêne. Physiol Vég 24, 307-314

Barnola $P$, Alatou D, Lacointe A, Lavarenne S (1990) Étude biologique et biochimique du déterminisme de la croissance rythmique du chêne pédonculé (Quercus robur L) Effets de l'ablation des feuilles. Ann Sci For 47, 619-631

Borchert R (1975) Endogenous shoot growth rhythms and indeterminate shoot growth in oak. Physiol Plant 35, 152-157

Brown CL (1971) Secondary growth. In: Trees structure and fonction (Zimmerman, Brown, eds) II, Springer-Verlag, New York, Heidelberg, Berlin, 67-123

Champagnat $P$ (1989) Rest and activity in vegetative buds of trees. Ann Sci For 46 suppl 9s-26s

Champagnat $P$, Allary S, Randier AM (1961) La ramification de la pousse herbacée du Charme et le problème des rameaux anticipés et des pousses de la St Jean. Mém Soc Bot Fr 108, 81-92

Champagnat $P$, Payan $E$, Champagnat $M, B a r-$ nola P, Lavarenne S, Bertholon C (1986) La croissance rythmique de jeunes chênes pédonculés cultivés en conditions contrôlées et uniformes. Naturalia Monspelliensia, Colloque International sur l'Arbre, 303-337

De Fay E (1986) Mode de croissance cambiale de quelques arbres tropicaux au stade juvénile. Naturalia Monspelliensia, Colloque International sur l'Arbre, 13-27

Dubroca E (1983) Évolution saisonnière des réserves dans un taillis de châtaignier, Castanea sativa Mill, avant et après coupe, Thèse $3^{e}$ cycle, Paris-Sud, 209 p

Greenwood M, Posnette AF (1950) The growth flushes of Cocoa. J Hortic Sci (Lond) 25 , 164-174

Greathouse DE, Laetsch WM, Phinney BO (1971) The shoot growth rhythm of a tropical tree, Theobroma cacao. Am J Bot 58, 281 286

Halle F, Martin R (1986) Étude de la croissance rythmique chez l'Hevea (Hevea brasiliencis Müll-Arg Euphorbiacées-Crotonoïdées). Adansonia 8, 475-503

Heide OM (1974) Growth and dormancy in Norway spruce ecotypes (Picea abies) I Interaction of photoperiod and temperature. Physiol Plant 30, 1-12

Holdsworth M (1963) Intermittent growth of the Mango tree. J West Afr Sci Assoc 7, 163-171

Klebs G (1914) Über das Treiben der einheimischen Baüme, speziell der Buche. Abh Heidelberg Akad der Wiss Math Naturwiss Klasse, 3, 1-116

Kramer PJ, Kozlowski TT (1979) Physiology of woody plants. Academic Press, New York, $811 p$

Lavarenne S, Champagnat $P$, Barnola $P$ (1971) Croissance rythmique de quelques végétaux 
ligneux de régions tempérées, cultivés en chambres climatisées à température élevée et constante et sous diverses photopériodes. Bull Soc Bot Fr 118,131-162

Lavender DP (1980) Effect of the environment upon the shoot growth of woody plants. In: Control of shoot growth in trees, Proc joint workshop IUFRO (Fredericton, Canada), 76. 106

Mialoundama $F$ (1985) Étude de la croissance rythmique chez le Gnetum africanum Welw. Thèse Doct État Orléans, $156 \mathrm{p}$

Nitsch JP (1957) Photoperiodism in woody plants. Proc Am Soc Hortic Sci 70, 526-544

Payan E (1982) Contribution à l'étude de la croissance rythmique chez de jeunes chênes pédonculés (Quercus pedunculata Ehrh). Thèse $3^{\ominus}$ cycle, Clermont-Ferrand II, $102 p$

Philipson R, Ward MW, Butterfield BG (1971) The vascular cambium, its periodic develop- ment and activity (Chapman \& Hall eds) Londres, $182 \mathrm{p}$

Scarrone $F$ (1969) Recherche sur les rythmes de croissance du Manguier et de quelques végétaux ligneux Malagasy. Thèse Doct État Sci, Clermont-Ferrand, $438 p$

Si-Mohamed C (1983) Germination; rythmes de croissance et morphogenèse de jeunes plants chez Castanea sativa Miller. Thèse Doctorat $3^{e}$ cycle,Clermont-Ferrand II, $200 \mathrm{p}$

Vilks $A$ (1974) Contribution à l'étude phytogéographique du département de la HauteVienne. Thèse Doctorat $3^{e}$ cycle, Toulouse, $127 \mathrm{p}$

Vogel M (1975) Recherche du déterminisme du rythme de croissance du Cacaoyer. Café Cacao Thé 14, 265-290

Wareing PF, Roberts DL (1956) Photoperiodic control of cambial activity in Rovinia pseudoacacia L. New Phytol 55, 356-366 